\title{
Assessment of Knowledge, Awareness and Attitude towards Oral Precancer and Cancer among Narikuravar Population in Pondicherry State
}

\author{
Sivaramakrishnan Muthanandam ${ }^{1}$ \\ Vezhavendhan N. ${ }^{1}$ Kishore M. ${ }^{4}$ \\ ${ }^{1}$ Department of Oral Pathology \& Microbiology, Indira Gandhi \\ Institute of Dental Sciences, Sri Balaji Vidyapeeth (Deemed to be) \\ University, Pondicherry, India \\ 2Socio-Behavioural \& Health Systems Research (SB\&HSR), Division, \\ Indian Council of Medical Research, New Delhi, India \\ ${ }^{3}$ Department of Periodontology, Indira Gandhi Institute of Dental \\ Sciences, Sri Balaji Vidyapeeth (Deemed to be) University, \\ Pondicherry, India \\ ${ }^{4}$ Indira Gandhi Institute of Dental Sciences, Sri Balaji Vidyapeeth \\ (Deemed to be) University, Pondicherry, India
}

\author{
Bontha V. Babu² Jananni Muthu ${ }^{3}$ Suganya R.
}

\begin{abstract}
Address for correspondence Dr. Jananni Muthu, MDS, Department of Periodontology, Indira Gandhi Institute of Dental Sciences, Sri Balaji Vidyapeeth (Deemed to be) University, Pondicherry, (e-mail: jannpearl@gmail.com).
\end{abstract}

South Asian J Cancer 2021;10:225-229.

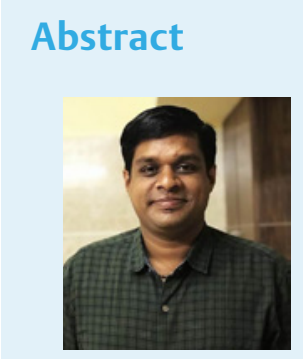

Sivaramakrishnan Muthanandam
Keywords

- Oral cancer

- awareness

- knowledge

- tribal population

- Narikuravar tribe
Objective Literature revealed that oral health status and awareness about oral hygiene measures of Narikuravar tribes were very poor. There was also an increased prevalence of tobacco usage among this population. Considering this, incidence and prevalence of oral precancerous and cancerous lesions are expected to be high in this population. Surprisingly, the literature search revealed a lack of data on the awareness, knowledge, and prevalence of oral cancer and precancer in this group. The primary objective of this survey is to assess knowledge, awareness and attitude toward oral precancer and cancer among the Narikuravar tribal population in Pondicherry state.

Materials and Methods This questionnaire survey is a part of the Model for Oral Cancer Eradication project conducted among the Narikuravar population in Pondicherry, which is funded by Indian Council of Medical Research (ICMR). A total of 153 participants were recruited for the survey. A prevalidated questionnaire was used for the survey.

Results The results indicated that $47 \%$ of the participants were aware of what is oral precancer and cancer. As much as $62 \%$ knew that chewing tobacco causes oral cancer and $44 \%$ thought that the growth of tissue in the mouth could be a sign of oral cancer. Almost $100 \%$ did not think that the presence of a red lesion, white lesion, or limitation of mouth opening could be signs of cancer or precancer. Only $16 \%$ thought that oral cancer is preventable.

Conclusion Awareness about oral cancer and precancer is inadequate among the Narikuravar population of Pondicherry. Majority of the population is not aware of the risks, signs, or treatment options of oral precancer and cancer. The National Programme for Prevention and Control of Cancer, Diabetes, Cardiovascular Diseases and Stroke (NPCDCS) identified the people's knowledge gaps, and improving awareness is one of the strategies of the program.
DOI https://doi.org/10.1055/s-0041-1733316 ISSN 2278-330X

How to cite this article: Muthanandam S, Babu B. V, Muthu J, et al. Assessment of Knowledge, Awareness and Attitude Toward Oral Precancer and Cancer among Narikuravar Population in Pondicherry State South Asian J Cancer 2021;10(4):225-229. (c) 2021. MedIntel Services Pvt Ltd.

This is an open access article published by Thieme under the terms of the Creative Commons Attribution-NonDerivative-NonCommercial-License, permitting copying and reproduction so long as the original work is given appropriate credit. Contents may not be used for commercial purposes, or adapted, remixed, transformed or built upon. (https://creativecommons.org/licenses/by-nc-nd/4.0/).

Thieme Medical and Scientific Publishers Private Ltd A-12, Second Floor, Sector -2, NOIDA -201301, India 


\section{Introduction}

Oral cancer indices and mortality show major differences worldwide, but the highest rates of incidence and prevalence are noted in India and neighboring countries, where it ranks among the top three types of cancer. ${ }^{1}$ The real challenge in the control of oral cancer lies in early diagnosis and prevention. Widening inequities in oral health status and treatment needs exist among different social groupings. ${ }^{2}$ Some ethnic groups live in isolation, even today, either geographically or socially with their traditional values and beliefs. They are commonly referred to as tribal population and considered to be the autochthonous people of the land. The Narikuravar tribal community is indigenous to Tamil Nadu state. They face common but consequential issues like poverty, illiteracy, communication problems, poor health care, and social discrimination. $^{3}$

An earlier study on oral health status and awareness about the oral hygiene measures of Narikuravar tribal population found that their oral health knowledge and status was very poor and needed greater attention of the dentists. ${ }^{4}$ This population also does not avail of oral health services as needed and tend to follow the traditional indigenous way of cure. One study reported that Narikuravar women had low rates of allopathic health care utilization, as they face stigma and discrimination while accessing health care. ${ }^{5}$

There was also wide long-term use of smokeless tobacco among both the genders. Considering this, the prevalence of cancerous or, at the least, precancerous lesions are expected to be high is this group. To our knowledge, only a few studies have assessed the oral health status of Narikuravar population in Tamil Nadu. In addition, there was poor knowledge and lack of awareness about oral health among the Narikuravar population in Tamil Nadu. ${ }^{4,6}$ However, these studies focused on oral hygiene and periodontal status and not the prevalence of oral cancer or knowledge of it. Thus, no studies are available on the prevalence of cancerous and precancerous lesions in the Narikuravar population. In lieu of the above, we proposed a study to explore a model for oral cancer screening and prevention among Narikuravar population in Pondicherry, and this research was supported by the Indian Council of Medical Research (ICMR). This paper presents a part of the findings from the first phase of the research project. This paper aims to assess the knowledge, awareness, and attitude of this population toward oral precancer and cancer.

\section{Materials and Methods}

This questionnaire survey is a part of a larger research project, Model for Oral Cancer Eradication, conducted among the Narikuravar population in Pondicherry. Prior permission from the local governing authority was also obtained for implementation of the research project. This research was approved by the institutional ethical committee. The Narikuravar population residing in the Narikuravar colony at Lawspet, Pondicherry, was considered for this study. This is the largest Narikuravar settlement in Pondicherry state.
A total of 153 participants in the settlement were recruited for the survey. Both male and female participants of age ranging between 15 to 70 years were selected randomly. Demographic details of the population is described in - Table 1. A prevalidated questionnaire was used for the survey. The questionnaire comprised items with a dichotomous response. The first question was: Do you know about oral cancer? Those who answered yes were taken through the rest of the survey. After completion of this survey in the initial phase, an awareness program regarding oral precancer and cancer was conducted for the population at their settlement. Informed consent was obtained from the participants of the study subjects after explaining to them about the aim of the study and methods employed. The entire survey was conducted by two trained dental specialists and a research assistant trained for the aforementioned project.

The willingness to participate was very less, and they had to be explained in detail about the importance and usefulness of the project. It took a lot of time to convince the people. In the initial phase, inauguration of the project was conducted and information regarding the project was provided; also doubts of the people were cleared by the principal investigator (S.M.).

Although their native language is Vargiboli, as they have long settled in Tamil Nadu, most of them are well versed in Tamil (the language of Tamil Nadu and Pondicherry). ${ }^{7}$ Hence, the survey was conducted in Tamil and their response was marked by the investigators. Only a few old people had difficulty in understanding the language, and we had a translator to facilitate the survey.

\section{Results}

Out of the 153 people included in the survey, 84 were men and 69 were women. Out of this, only 72 (47\%) answered positively for the first question. It indicated that only $47 \%$ of participants are aware of oral cancer. These participants were taken through the rest of the survey. With regard to

Table 1 Demographic detail of the population

\begin{tabular}{|c|c|c|}
\hline \multicolumn{3}{|l|}{ Gender } \\
\hline Male & 154 & 46.8 \\
\hline Female & 175 & 53.2 \\
\hline \multicolumn{3}{|l|}{ Age } \\
\hline $18-30$ & 97 & 29.4 \\
\hline $31-60$ & 129 & 39.2 \\
\hline 61 and above & 103 & 31.4 \\
\hline \multicolumn{3}{|l|}{ Educational status } \\
\hline Uneducated & 300 & 91 \\
\hline $\begin{array}{l}\text { Completed primary } \\
\text { school }\end{array}$ & 24 & 8.3 \\
\hline $\begin{array}{l}\text { Completed middle } \\
\text { school }\end{array}$ & 2 & 0.6 \\
\hline Completed high school & 0 & 0 \\
\hline Basic degree & 3 & 0.1 \\
\hline
\end{tabular}


the question on cause oral cancer or precancer, 49 (32\%) participants answered that smoking causes cancer, 45 (29.4\%) participants attributed it to chewing tobacco, and only 21 (13.7\%) participants reported that consumption of alcohol causes oral cancer. For the question what they thought might be oral cancer, 32 (20.9\%) participants answered that it is the growth of tissue, 23 (15\%) participants thought that ulcer might be cancer, and only one participant opined that red color change in the mouth is oral cancer. No one reported that white color change, or reduced mouth opening could be a sign of oral cancer or precancer. Only $15(9.8 \%)$ participants agreed that oral cancer could kill. Only 12 (7.8\%) participants thought that oral cancer is preventable and 45 (29.4\%) participants, who were aware of oral cancer, possessed no knowledge on prevention. Further probing was carried out to determine whether they knew oral cancer treatment is possible if detected early. Only $22(14.4 \%)$ answered positively with regard to treatment, and the remaining participants did not even know that oral cancer is treatable if it is detected early. Of the total participants, 27 (17.6\%) participants thought medicines would cure oral cancer, 12 (7.8\%) participants thought surgery is needed, and 4 (2.6\%) participants thought both are necessary as a treatment modality for oral cancer. Further, the participants were asked whether they liked to know more about oral cancer, and all participants expressed that they are willing ( - Table 2 ).

\section{Discussion}

In India, 104 million tribal people reside, constituting 8.6\% of the total Indian population. It is estimated that about 30,000 Narikuravars live in Tamil Nadu, and some live in the neighboring Pondicherry state. The main occupation of the tribe involves hunting small animals for living. However, a ban is imposed on the same by the government. Hence, they have taken to other occupations such as selling beads, toys, etc. in markets, bus stands, fairs, etc., and leading a nomadic lifestyle where their children never get the opportunity to be educated. ${ }^{8}$ But the population selected for this study lives in a settlement allotted to them by the government of Pondicherry. Although they tend to stay away from home for few days by engaging themselves in selling things, they eventually come back to the settlement.

The major issues pressing this society are illiteracy, poverty, and social discrimination. Most of them live in extreme exteriors of civilization and very difficult to locate. ${ }^{9}$ The population in our study also lives in a settlement that is located 15 kilometers outside the city limits. Poor socioeconomic status, geographical isolation, and their discernment prevent the people from availing health care services. This led them to depend on indigenous self-health care practices. Most of them do not avail allopathic services to address chronic or nonserious illnesses. ${ }^{10}$ It was also found that they lack

Table 2 Frequency distribution of the responses for the items in the questionnaire

\begin{tabular}{|c|c|c|c|}
\hline & Yes & No & \\
\hline Do you know about oral cancer/precancer? & $72(47.05 \%)$ & \multicolumn{2}{|l|}{$81(52.9 \%)$} \\
\hline \multicolumn{4}{|c|}{ Which of the following do you think causes oral cancer/precancer? } \\
\hline Smoking & $49(68.05 \%)$ & \multicolumn{2}{|l|}{$23(31.9 \%)$} \\
\hline Chewing tobacco & $45(62.5 \%)$ & \multicolumn{2}{|l|}{$27(37.5 \%)$} \\
\hline Alcohol & $21(29.15 \%)$ & \multicolumn{2}{|l|}{$51(70.83 \%)$} \\
\hline \multicolumn{4}{|c|}{ Which of the following do you think is oral cancer/precancer? } \\
\hline & Yes & No & Don’t know \\
\hline Growth of tissue & $32(44.44 \%)$ & $34(47.22 \%)$ & $12(16.67 \%)$ \\
\hline Ulcer & $23(31.94 \%)$ & $7(9.72 \%)$ & $42(58.33 \%)$ \\
\hline Red color change & $1(1.39 \%)$ & $71(98.61 \%)$ & $0(0 \%)$ \\
\hline White color change & $0(0 \%)$ & $72(100 \%)$ & $0(0 \%)$ \\
\hline Reduced mouth opening & $0(0 \%)$ & $72(100 \%)$ & $0(0 \%)$ \\
\hline \multicolumn{4}{|l|}{ What do you think about the following statements? } \\
\hline Oral cancer can kill & $15(20.84 \%)$ & $19(26.39 \%)$ & $38(52.78 \%)$ \\
\hline Oral cancer prevention is possible & $12(16.67 \%)$ & $45(62.5 \%)$ & $15(20.84 \%)$ \\
\hline Oral cancer treatment is possible if detected early & $22(30.56 \%)$ & $12(16.67 \%)$ & $38(52.78 \%)$ \\
\hline \multicolumn{4}{|c|}{ Which of the following do you think are the treatment for oral cancer? } \\
\hline Medicines & $27(37.5 \%)$ & $4(5.56 \%)$ & $41(56.94 \%)$ \\
\hline Surgery & $12(16.67 \%)$ & $14(19.45 \%)$ & $46(63.89 \%)$ \\
\hline Both & $4(5.56 \%)$ & $5(6.94 \%)$ & $63(87.5 \%)$ \\
\hline Do you like to know more about oral cancer? & $153(100 \%)$ & $0(0 \%)$ & \\
\hline
\end{tabular}


sufficient knowledge of the medical system, access to facilities and medical staff and appropriate health insurance scheme that would alleviate the financial burden of health care. ${ }^{8}$ But another study on women of this tribe revealed that women preferred allopathic care to traditional practices for pregnancy and related issues. ${ }^{11}$

Studies assessing their oral health, awareness, and practices are very less. ${ }^{4,6}$ Selvakumar assessed the oral health status of the Narikuravar tribe in Tirunelveli district of Tamil Nadu. ${ }^{4} \mathrm{He}$ observed poor knowledge and lack of awareness about oral health among these people. Dhivya et al evaluated the accessibility and barriers to oral health and the oral health status of the Narikuravar population in Chennai. They found an increased prevalence of dental caries and periodontal problem in the population, and the population tended to markedly neglect oral health. ${ }^{6}$

Another important issue is that about $60 \%$ of the population used tobacco products. ${ }^{6}$ Prabhu et al reported that $57 \%$ of the study population use either smoking or smokeless tobacco. Consuming packaged tobacco prepared by using tobacco leaves, areca nut, and slaked lime was the most prevalent practice in the study area. The study also revealed their willingness to quit the habit which was not successful without professional assistance. ${ }^{12}$ Considering these facts, prevalence of oral cancerous and precancerous lesions are expected to be high in this population. The literature revealed no data regarding the knowledge, attitude, and prevalence of oral precancer and cancer among this population.

In lieu of the abovementioned facts, in that the population is discriminated against when it comes to availing of regular health services, neglects oral health, and consumes tobacco at a high rate, a project to design a model to screen and prevent oral cancer was initiated with the support of the ICMR. The current survey is the baseline on people's knowledge, awareness, and attitude toward oral precancer and cancer. The present study reveals that only $47 \%$ knew what is oral precancer and cancer. The rest of the population is unaware of oral cancer. This shows that the population is highly unaware of the disease. This is in accordance with results revealed by Selvakumar and Dhivya et al, in which they concluded that the Narikuravar population lacked knowledge about oral health. ${ }^{4,6}$ Out of the population who was aware of oral cancer, $62 \%$ knew that chewing tobacco causes oral cancer, but still most of them were chewing tobacco. Only $44 \%$ thought that the growth of tissue in the mouth could be a sign of oral cancer, and 31\% thought that an ulcer might be a sign of oral cancer. Almost 100\% did not think that the presence of a red lesion, white lesion, or limitation of mouth opening could be a sign of cancer or precancer. In a study done by Prabhu et al, $86.2 \%$ knew about the oral hazards of tobacco, but only $16.9 \%$ were particularly aware that chewing tobacco products could cause oral cancer. ${ }^{12}$

A small proportion of participants were aware that oral cancer can kill, and oral cancer prevention is possible. Thus, majority of the participants possessed poor knowledge on oral cancer, its signs, causes, treatment and prevention. The National Programme for Prevention and Control of Cancer, Diabetes, Cardiovascular Diseases and Stroke (NPCDCS) identified the gaps in the knowledge of the people, and awareness generation and promotion of healthy lifestyle is one of the program's strategies. ${ }^{13}$ Awareness generation about warning signs of cancer is one of the health promotion activities of the NPCDCS. Studies of this kind are very useful in identifying high-risk populations who possess poor awareness of cancers.

\section{Conclusion}

From the findings of this study, it is clear that awareness about oral cancer and precancer is inadequate among the Narikuravar tribal population of Pondicherry. Majority of the participants are not aware of the risks, signs or treatment options of oral precancer and cancer. Given their habits and their poor knowledge about oral cancer and precancer, a targeted and community-based approach is very essential to change the knowledge, attitude, and behaviour of these people. The study has implications with reference to NPCDCS.

Source (s) of Support

The study was funded by ICMR. Project IDN: 2019 - 3629

Conflict of Interest

None declared.

Acknowledgement

Indian Council of Medical Research (ICMR), Government of India.

\section{References}

1 Sankaranarayanan R, Ramadas K, Thomas G, et al. Trivandrum Oral Cancer Screening Study Group. Effect of screening on oral cancer mortality in Kerala, India: a cluster-randomised controlled trial. Lancet 2005;365(9475):1927-1933

2 Patrick DL, Lee RS, Nucci M, Grembowski D, Jolles CZ, Milgrom P. Reducing oral health disparities: a focus on social and cultural determinants. BMC Oral Health 2006; 6(Suppl 1):S4

3 Atheeque M, Nishanthi R. Explanatory analysis of life style of Narikurava community in Pudukkottai district. International Journal of Advanced Research and Development 2016;1:21-24

4 Kumar S. To assess the oral health status and awareness about the oral hygiene measures of the jackal people (Narikuravar tribes) - A cross sectional study. Int J Curr Res 2017;9:50720-50723

5 Zafiu M; Joseph Wharton Scholars. Health Access for Vulnerable Groups: A Study on the "Gypsy" Narikuravar Community in Tamil Nadu, India. Available at: http://repository.upenn.edu/joseph_wharton_scholars/36. Accessed May 5, 2021

6 Dhivya LN, Prabhu D, Raj Mohan, et al. Accessibility and barriers to oral health care among gypsy tribes in Chennai: a cross sectional study. Indian J Public Health Res Dev 2019;10:233-237

7 Mathai EK, Vijayakumar K; SIL International. A Sociolinguistic Survey among the Vaagri Booli Speakers of South India. Available at: https://www.sil.org/system/ files/reapdata/ 15/ 02/66/150266533956350183149970295715866453851/ silesr2018_005.pdf. Accessed May 5, 2021

8 Jayachitra J. Life style and major issues pertaining to the Narikuravar (Nomads) Community in Tamil Nadu. Ind J Res 2016;5:412-413 
9 Dragoir CL, Zafiu M. Vulnerable populations' access to health care: a study of the nomadic 'gypsy' Narikuravars in Tamil Nadu, India. International Journal of Roma Studies 2019;1:58-83

10 Jayachitra J. A study on Narikuravar (Nomads) community in Palamalai Nagar, Sivagangai district, Tamilnadu. GJRA - Glob J Res Anal 2016;5:28-30

11 Alex G, Folk healing and the negotiation of shifting social identities in Tamil Nadu, India. MMG Working Paper, Munich, Germany: Max Planck Institute for the Study of Religious and Ethnic Diversity; 2009; Retrieved from http://www.mmg.mpg.
de/fileadmin/user_upload/documents/wp/WP_09-05_Alex_ Folk-healing.pdf

12 Prabhu D, Nirmala S, Aurlene N. Tobacco menace and challenges in quitting tobacco in an indigenous population of Tamil Nadu: a cross-sectional survey. J Clin Diagn Res 2019;13(4):ZC15-ZC20

13 Government of India. National Programme for Prevention and Control of Cancer, Diabetes, Cardiovascular Diseases and Stroke (NPCDCS). Available at: http://www.nrhmhp.gov.in/ sites/default/files/files/NCD_Guidelines.pdf. Accessed May 5, 2021Photo To Come 